\title{
Diachronie, histoire de la langue : synthèse et perspectives
}

\author{
Sophie Prévost \\ Lattice UMR 8094 (CNRS / ENS Paris)
}

Cette brève contribution a pour but de donner un aperçu du paysage dessiné par les contributions retenues dans la thématique « Diachronie, histoire de la langue » du CMLF. Elle sera présentée dans le cadre de la Table Ronde, son but étant autant d'effectuer la synthèse des communications qui auront été présentées que de lancer la discussion sur les perspectives de notre discipline.

\section{Données générales : évaluations quantitative et thématique}

Il a été proposé dans la section «Diachronie, histoire de la langue » 26 communications, ce qui situe notre thématique dans le peloton du milieu. Parmi elles, 6 l'étaient aussi dans une autre thématique (lexique(s) / linguistique du texte et de l'écrit, stylistique/ sémantique). Nous en avons retenu 15 (dont 2 qui avaient également postulé, en sémantique pour l'une, en linguistique du texte et de l'écrit pour l'autre).

Le domaine de l'histoire de la langue présente une spécificité qui le distingue de l'ensemble des autres domaines de la linguistique, puisque d'une certaine manière il les comprend tous, ou, plus justement, il leur est orthogonal. Une approche diachronique peut s'intéresser aux aspects morphologiques, sémantiques, pragmatiques... de même que la dimension phonétique, syntaxique,... de tel ou tel phénomène peut être envisagée dans une perspective diachronique. La démarche historique n'en comporte pas moins ses caractéristiques propres, du fait même qu'elle appréhende les faits dans leur évolution, ce qui suppose a priori une théorie ou au moins un cadre d'analyse spécifique.

Il semblait intéressant de mettre en relation la représentation plus ou moins forte des différents domaines, parmi les propositions retenues dans la thématique «Diachronie, histoire de la langue », avec la représentation plus ou moins importante des différentes thématiques au sein du CMLF ${ }^{1}$. On pouvait en effet se demander s'il y aurait convergence ou au contraire divergence, si les domaines privilégiés dans le cadre des approche historiques reflèteraient ceux qui le sont dans l'ensemble du CMLF.

La réponse est quelque peu complexe, dans la mesure où certaines communications retenues ${ }^{2}$ en «Diachronie, histoire de la langue » se trouvent à la croisée de plusieurs domaines. C'est le cas pour 4 d'entre elles : 2 relèvent à la fois de la linguistique du texte et, pour l'une de la syntaxe, pour l'autre de la phonétique, tandis que deux autres relèvent de la sémantique et, pour l'une de la morphologie, pour l'autre du lexique ${ }^{3}$. Au-delà de ces communications «mixtes», on voit se dégager quelques tendances nettes : les thématiques qui sont fortement représentées ou au contraire peu illustrées dans l'ensemble du CMLF le sont généralement pareillement au sein de la section Diachronie. Ainsi, alors que 22 communications ont été retenues dans la thématique "Sémantique » (sur 42 soumises), on dénombre 6 communications relevant prioritairement de ce domaine dans la section «Diachronie, histoire de la langue ", et 2 qui en relèvent de façon secondaire. Les communications touchant de près à la sémantique représentent donc la moitié de celles proposées dans notre section. A l'inverse, les sections «Morphologie » et «Phonétique, phonologie et interfaces» ne dénombrent que 5 communications chacune (sur 7 et 10 soumises repsectivement), désaffection relative qui rejoint celle que l'on peut observer dans la section «Diachronie, histoire de la langue »: une seule communication s'inscrit pleinement dans le domaine de la phonétique, une autre en relève partiellement, tandis que la morphologie n'est véritablement abordée que dans une communication. La syntaxe constitue en revanche une exception remarquable, puisqu'elle est représentée par 21 communications (sur 28 soumises) au sein de la section « Syntaxe », mais seulement par 2 communications dans notre section, l'une portant sur les subordonnées asyndétiques, l'autre sur les constructions détachées en rupture. 
Il serait probablement hasardeux de considérer que les communications retenues dans la section «Diachronie, histoire de la langue » dessinent l'exacte cartographie actuelle de l'activité au sein du domaine dans le monde. Elles n'en constituent pas moins un certain reflet.

Précisons pour clore cette présentation générale que si la France est, de manière attendue, bien représentée parmi les auteurs, les communications issues d'autres pays constituent la moitié de l'ensemble. On dénombre ainsi 7 auteurs pour la France, 2 pour la Belgique, 2 pour le Canada (Québec), 1 pour la Tunisie, 1 pour l'Allemagne et 1 pour le Japon. Pour ne pas déséquilibrer cette parfaite répartition (7 auteurs français contre 7 étrangers), le hasard (permis par l'anonymat des papiers soumis) nous a fait retenir un couple franco-belge !

Nous reviendrons plus en détails en 4. sur le contenu des propositions, mais avant cela il convient de dire quelques mots sur la place faite, d'une part à la perspective diachronique, d'autre part aux corpus.

\section{Diachronie et synchronie}

Il semble qu'il existe encore, dans l'esprit de certains, une relation quasi bi-univoque entre « diachronie » et "langue ancienne », au sens où le fait de s'intéresser au français médiéval engendrerait souvent une démarche diachronique, et surtout qu'une approche diachronique ne pourrait concerner que des états de langues anciens. On sait que c'est inexact : d'une part, nombreuses sont les études portant sur l'ancien ou le moyen français qui adoptent une perspective ouvertement synchronique, d'autre part il est tout à fait légitime - et même fortement recommandé - de se pencher sur les évolutions récentes de certains phénomènes linguistiques, et même sur une période brève, si les changements intervenus se sont produits dans un intervalle réduit. En témoigne par exemple l'évolution récente et assez rapide de l'adverbe trop, en train de passer, chez de nombreux locuteurs, d'une valeur de marqueur du degré excessif à celle de simple marqueur de haut degré ${ }^{4}$.

Il n'en demeure pas moins vrai que le fait de considérer des états de langue anciens permet d'évaluer avec plus de recul, et partant davantage d'objectivité, l'évolution qu'a pu connaître tel ou tel fait linguistique. La distance permet aussi - et c'est capital- de juger si l'évolution est terminée, ou au moins connaît une pause durable, ce qui est évidemment plus difficile à estimer lorsque l'on s'attache à une période récente. On sait en effet que les changements ont tous leur propre temporalité, et l'on ne peut donc se fier à l'évolution antérieure d'un phénomène analogue pour préjuger de la durée de l'évolution du phénomène considéré. Ces différentes raisons expliquent probablement que, à l'image des études diachroniques en général, celles proposées ici ne vont pas au-delà du $19^{\text {ème }}$ siècle, ce qui nous donne cependant une large chronologie, puisque l'une des études s'intéresse à l'époque du proto-français !

En toute rigueur, une démarche véritablement diachronique ne se réduit pas à la prise en compte d'états synchroniques successifs. Elle doit expliquer comment l'on passe de l'un à l'autre. Il ne suffit pas d'enregistrer les changements, il faut rendre compte de l'évolution ${ }^{5}$. La tâche n'est pas simple, on le sait.

La très grande majorité des communications retenues s'inscrit dans une perspective diachronique. Il en est 3 pour lesquelles cette dimension est moins perceptible. Dans la mesure où elles présentaient un intérêt scientifique évident et qu'elles esquissaient quand même une mise en perspective temporelle, il a été décidé de les retenir.

Que les communications envisagent l'évolution d'un phénomène en continu sur une certaine durée, plus ou moins longue, comme c'est le cas pour la majorité, ou bien qu'elles mettent en perspective deux époques différentes, on observe toujours un effort pour saisir, comprendre et restituer les ressorts et les mécanismes du changement. De manière intéressante, l'une des études propose même la mise en regard de l'évolution de deux types de marqueurs analogues (marqueurs de focalisation), l'une en latin tardif, l'autre en moyen français, comparaison qui met nettement au jour les points communs entre les deux phénomènes. 


\section{Place aux corpus!}

Travailler sur une langue ancienne et / ou chercher à rendre compte de l'évolution d'un phénomène linguistique suppose de s'appuyer sur des énoncés attestés. Pour ce qui est des états de langue anciens, le locuteur ne possède en effet pas de compétence et il ne peut faire appel à des informateurs qui en seraient dotés. Quant à travailler dans une perspective diachronique, quelle que soit l'époque, il nous semble pour le moins hasardeux d'envisager de le faire à partir d'énoncés forgés. Bref dans et l'autre cas, et a fortiori quand les deux sont réunis, les «textes », au sens large du terme, sont indispensables. Pendant longtemps ils n'ont cependant très souvent servi que de réservoirs à exemples. La numérisation croissante des textes, ainsi que le développement des outils pour extraire et traiter les données pertinentes ont grandement facilité les tâches. Il est désormais possible de s'appuyer sur des corpus de plusieurs millions d'occurrences, si tant est que l'on possède les forces, non seulement pour en extraire les données recherchées, mais aussi pour les traiter ensuite (on est parfois submergé par l'avalanche de données...).

Pour une très large majorité, les communications présentées font état d'une démarche raisonnée sur corpus. Celle-ci revêt cependant différentes formes. Certaines études s'appuient ainsi sur les quelques bases textuelles existantes, principalement, la Base de Français Médiéval (BFM) pour l'ancien français (ICAR - CNRS / ENS-LSH Lyon), la base du Dictionnaire de Moyen Française (DMF) pour le moyen français (ATILF - CNRS / université de Nancy), et la base de Frantext pour les siècles qui suivent (idem). D'autres études, centrées sur des phénomènes, ou bien fort nombreux, ou bien complexes à extraire ou longs à analyser, restreignent leur champ d'investigation à quelques textes, bien identifiés. Il s'agit en majorité d'éditions critiques, mais aussi parfois d'éditions diplomatiques (ainsi des deux études, portant pour l'une sur des chartes écrites à Liège avant 1292, pour l'autre sur des actes champoenois méridoniaux de 1230 à 1300). Enfin, deux communications s'appuient sur des sources lexicographiques. Plusieurs auteurs étaient leur étude sur des données quantifiées précises.

\section{Données empiriques et approches théoriques : quelques tendances}

Nous ne résumerons pas ici l'ensemble des communications : leur présence dans ce même volume et leur présentation orale dans le cadre du colloque ne justifie guère l'exercice, et le résumé, partiel et possiblement partial, resterait bien pâle à côté de l'original.

On essaiera en revanche de dégager quelques tendances parmi l'ensemble des communications présentées. Rappelons tout d'abord que, si la sémantique est le domaine le mieux représenté dans notre section (à l'image de l'ensemble du CMLF), l'ensemble des autres domaines l'est aussi, dans des proportions certes variables, ainsi que nous l'avons évoqué en 1. Deux communications explorent la dimension de l'écrit du texte, l'une en se penchant sur l'interaction entre ponctuation et syntaxe, l'autre à travers la mise en relation des graphies et de la prononciation. Le domaine de la prononciation est également abordé à travers la question de l'origine de l'accent en proto-français. La dimension lexicale est quant à elle explorée à travers deux communications, l'une qui, en se penchant sur la norme lexicale au $19^{\text {ème }}$ siècle, introduit une dimension socio-linguistique, l'autre qui, tout en s'appuyant sur des données lexicographiques, se situe ouvertement dans le cadre de la pragmaticalisation.

Parmi les études qui s'inscrivent plus ou moins pleinement dans le champ de la sémantique, toutes s'attachent à l'évolution d'une expression (ains, vouloir citationnel, car, selon X...), ou à un groupe d'expressions dans le cadre d'une approche sémantico-référentielle (ainsi des marqueurs de focalisation, ou bien des marqueurs de continuité référentielle), ou morpho-sémantique (préverbes). Il est significatif que la très grande majorité d'entre elles sollicite l'une des notions suivantes: grammaticalisation, lexicalisation, pragmaticalisation, subjectification, mais aussi métonymie et métaphore (ainsi de l'étude de vouloir citationnel). Il s'agit de concepts qui se sont développés relativement récemment pour certains (ainsi de la pragmaticalisation, qui a entre autres permis de réduire et de mieux circonscrire le domaine de la grammaticalisation), et en partielle relation entre eux : la subjectification intervient souvent dans le cadre de la grammaticalisation, mais elle peut aussi se manifester indépendamment. Il en est de même de la métaphore et de la métonymie, concepts fort anciens, mais qui ont connu depuis une vingtaine 
d'années un développement spécifique dans le cadre de la grammaticalisation. Il apparaît que cette dernière, qu'elle soit envisagée comme un type de phénomène ou comme une théorie, demeure un cadre privilégié pour penser et analyser bon nombre de phénomènes de changements.

Quelques-unes des communications, prioritairement descriptives, ne se situent pas dans un cadre théorique explicite. Elles n'en sont pas moins d'une grande qualité, et mettent au jour des faits inédits ou renouvellent l'approche de certains autres. L'évolution d'un fait ne se laisse pas toujours appréhender directement dans un cadre théorique, il faut parfois la mise en relation avec d'autres faits pour que l'évolution devienne signifiante ${ }^{6}$. De ce point de vue, la disparition des formes est assez instructive : comme le montre la communication consacrée à cette question, la prise en compte de plusieurs cas de ce type prouve qu'il ne s'agit nullement d'épiphénomènes mais au contraire de l'émergence de nouveaux paramètres.

\section{Quelles perspectives pour la diachronie du français ?}

Nous souhaitons que la discussion qui aura lieu dans le cadre de la Table Ronde puisse fournir des éléments de réponse à cette vaste question. Une chose est sûre : les études sur l'histoire de la langue, qu'il s'agisse d'ailleurs du français ou d'autres langues, connaissent depuis plusieurs années un net (re)gain d'intérêt. Celui-ci s'observe chez de jeunes collègues qui entreprennent de se lancer dans ce vaste champ d'étude où beaucoup encore reste à défrîcher. On constate par ailleurs que des collègues travaillant en synchronie sur des états de langue contemporains manifestent une curiositié croissante envers les états de langue anciens, étant de plus en plus nombreux à se pencher sur l'évolution passée des phénomènes qu'ils cherchent à décrire et à expliquer en synchronie. On le sait : si une approche diachronique améliore notre connaissance des états passés (plus ou moins reculés) d'une langue, elle permet aussi une meilleure compréhension de la langue que nous parlons et écrivons aujourd'hui. On peut même considérer qu'elle autorise dans une certaine mesure la formulation d'hypothèses quant à son devenir, et au-delà, comme un idéal vers lequel nous tendons, une relative compréhension du langage en tant que faculté cognitive (cela grâce à la comparaison de l'évolution des différentes langues).

Assurément l'histoire du français a de beaux jours devant elle : elle suscite l'enthousiasme, renferme encore, sur 12 siècles, de nombreux aspects peu ou pas explorés, et elle bénéficie depuis quelques décennies d'un renouveau théorique capital.

Souhaitons que la discussion qui clôturera la présentation des différentes communications de notre section saura mettre au jour, d'une part les éventuelles faiblesses que peut connaître notre discipline, et d'autre part, mieux que nous ne l'avons fait ici, les apports récents et les pistes à suivre, tant du point de vue de l'exploration des données empiriques que des approches théoriques.

\footnotetext{
${ }^{1}$ Rappelons que le CMLF regroupe, outre la section « Diachronie, histoire de la langue », 11 thématiques, et que les études menées dans 9 d'entre elles sont susceptibles de s'inscrire dans une perspective diachronique. Cela semble en revanche plus difficile pour les thématiques «Didactique» et "Psycholinguistique, acquisition », l'une et l'autre étant des disciplines relativement jeunes. La mis ene oeuvre d'une dimension diachronique supposerait de réduire celle-ci à un intervalle temporel assez bref, et récent.

${ }^{2}$ Nous n'avons en effet considéré que les propositions retenues.

${ }^{3}$ Certes, plusieurs autres communications relèvent aussi dans une certaine mesure de différentes thématiques, ce qui n'est guère étonnant dans la mesure où la description d'un phénomène linguistique suppose souvent d'en considérer différentes dimensions, a fortiori lorsqu'il s'agit de rendre compte de son évolution. On peut cependant aisément repérer, pour ces communications, un angle d'approche dominant.

${ }^{4}$ Il est cependant vrai que cette dernière valeur existait déjà en ancien français. Il semble qu'elle ait connu une longue éclipse, ou au moins période de mise au second plan, avant de revenir assez récemment au premier plan.
} 
ISBN 978-2-7598-0358-3, Paris, 2008, Institut de Linguistique Française

Diachronie, histoire de la langue

DOI $10.1051 / \mathrm{cmlf0} 321$

\footnotetext{
${ }^{5}$ Voir C. Marchello-Nizia (1995) L'évolution du français : ordre des mots, démonstratifs, accent tonique. Paris : A. Colin, pp. 28-29.

${ }^{6}$ Voir à ce propos C. Marchello-Nizia (2001) «Grammaticalisation et évolution des systèmes grammaticaux », Langue française 130, pp. 33-41, et C. Marchello-Nizia (2006) Grammaticalisation et changement linguistique, Bruxelles, De Boeck.
} 